\title{
Redox status in benign prostatic hyperplasia and non-metastatic prostate cancer in the Algerian population
}

\author{
Type of article: Conference abstract \\ Rima Mouhoub ${ }^{1}$, Karima Haddad ${ }^{1}$, Sarah Goumeri ${ }^{1}$, Abdel Aziz Chibane ${ }^{2}$, Raouf \\ Oucherif ${ }^{2}$, Bahia Djerdjouri ${ }^{1}$, Malika Khelii ${ }^{1}$ \\ 1. Department of Cellular and Molecular Biology, Faculty of Biological Sciences, \\ University of Science \& Technology Houari Boumediene, USTHB ,Beb Ezzouar, Algiers, \\ Algeria. \\ 2. Department of Urology, Medical University Hospital of Mustapha Bacha, Algiers, \\ Algeria.
}

mouhoub.rima@gmail.com

\begin{abstract}
Background: Depletion of cellular antioxidants can result from free radical formation due to normal endogenous reactions and the ingestion of exogenous substances and environmental factors. The levels of reactive oxygen species (ROS) have been shown to be significantly altered in malignant cells and in primary cancer tissues. We undertook the present study to investigate the possible alteration of oxidant/antioxidant status in Algerian patients with benign prostatic hyperplasia $(\mathrm{BPH})$ and prostate cancer $(\mathrm{PCa})$.

Methods: In total, 89 subjects made up of 26 patients with non-metastatic prostate cancer, 31 with benign prostatic hyperplasia (BPH), and 32 controls participated in this study. The concentrations of plasmatic malondialdehyde (MDA), erythrocytes catalase activity (CAT), and the plasma glutathione levels (GSH) were estimated using standard procedures.

Results: The results showed that MDA concentrations were significantly increased while erythrocyte catalase activity was significantly decreased in the prostate cancer group versus controls $(P<0.01)$ and BPH group $(P<0.05)$. GSH levels were lowered in prostate cancer patients versus control group with no significant changes.

Conclusions: Our results suggest that an alteration in the lipid peroxidation index with concomitant changes in the antioxidant defense system in prostate cancer patients compared with controls. We hypothesize that an altered pro-oxidantantioxidant balance may lead to an increase in oxidative damage and consequently may play an important role in prostate carcinogenesis.
\end{abstract}

Keywords: Prostate cancer (PCa), Benign Prostatic Hyperplasia (BPH), Glutathione (GSH), Malondialdehyde (MDA), Catalase activity (CAT). 


\section{Declaration of conflicts}

This article was selected from the International Conference of Health Sciences and Medical Technologies 2016 ICHSMT'16 abstracts book.

\section{Authors' biography}

No Biography

\section{References}

No references 